\title{
Simulation of optical properties of semiconductor multilayers from extreme ultraviolet to far infrared
}

\begin{abstract}
Optical properties of semiconductors play a critical role in various applications including the design and manufacture of optical components, devices \& sources, energy conversion and process monitoring \& control. While the fundamental understanding of the optical properties of semiconductors has grown over the years, reliable data of the optical constants of semiconductors, particularly in the infrared range of wavelengths, is severely lacking in the literature. In this overview, detailed case studies of the optical properties of Silicon on Insulator (SOI) and Ge photodetectors, based on Forouhi-Bloomer dispersion equation, as function of photon energy (or wavelength) and thickness are presented. The obtained simulation results, based on this relation, are in good accord with the literature values and are consistent with some well-accepted studies. Furthermore, the results reported in this analysis are helpful for the determination and realization of the optical response of materials under conditions of varying photon energy and thickness.
\end{abstract}

Volume 4 Issue 5 - 2020

\author{
Leqi Lin,' Ravindra NM² \\ 'Department of Civil and Environmental Engineering, New \\ Jersey Institute of Technology, USA \\ Interdisciplinary Program in Materials Science \& Engineering, \\ New Jersey Institute of Technology, USA
}

Correspondence: Ravindra NM, Interdisciplinary Program in Materials Science and Engineering New Jersey Institute of Technology, Newark, New Jersey 071 02, USA, Tel + I 973596 3278/6453, Email n.m.ravindra@njit.edu,nmravindra@gmail.com

Received: October 07, 2020 | Published: October 21, 2020

\section{Introduction}

Optical properties of semiconductors play a critical role in various applications including the design and manufacture of optical components \& devices such as detectors, filters, imagers, lenses, optical coatings, photonic crystals, sensors and waveguides, sources such as lasers \& light emitting diodes, energy conversion, process monitoring \& control using in situ, non-contact techniques such as radiation pyrometry, spectroscopic ellipsometry and time resolved reflectivity. While the fundamental understanding of the optical properties of semiconductors has grown over the years, reliable data of the optical constants of semiconductors, particularly in the infrared range of wavelengths, is severely lacking in the literature. In order to meet the unprecedented demands for advanced highspeed semiconductor devices, silicon-based materials such as siliconon-insulator (SOI) and silicon on sapphire (SOS) substrates have attracted considerable interest due to significant transformation of their optical and electrical properties caused by quantum-confinement effects. Silicon is an excellent material system for optical confinement and wave transmission in the near infrared range ${ }^{1}$ and $\mathrm{SiO}_{2}$ has good compatibility with the existing CMOS technology for microelectronics and photonics. ${ }^{2}$ High quality Ge crystals have been used for highly sensitive near-infrared (NIR) photodetectors in the radiation wavelength of $1300-1600 \mathrm{~nm},{ }^{3}$ and exhibit significant absorption in the telecommunication wavelengths range. ${ }^{4}$ Ge photodetectors are the first active optoelectronic devices monolithically integrated on $\mathrm{Si}^{5}$ and have revolutionized optical communications. Their performance is comparable to III-V semiconductor detectors (e.g., InGaAs) due to their shorter absorption lengths, higher electron mobilities, faster response times and lower power dissipation. ${ }^{3}$

Modeling the radiative properties of semiconductor multilayers such as SOI structures and Ge photodetectors is important in several areas of optoelectronics in the desired near-infrared (NIR) range of wavelengths. These material structures are used in light detectors, waveguides and other optical applications. Optical dispersion depends on the incident photon energy $(E)$ or wavelength of light $(\lambda)$, as well as on the material properties and the device fabrication conditions. Optical properties can be expressed as the real and imaginary parts of the complex index of refraction relation as $N=n-i k$, where $n$ is the refractive index and $k$ is the extinction coefficient. Optical functions, $n$ and $k$, are intrinsic material properties and may be thought of as the fingerprint of a material, and cannot be measured directly. ${ }^{6}$ They are determined from extrinsic measurements that depend on their surface morphology, dopant type and dopant concentration/resistivity etc. (e.g., reflectance and transmittance, or ellipsometric parameters). In this study, an investigation of the optical properties of semiconductors, based on the Forouhi-Bloomer dispersion equations for $n$ and $k$, is presented by utilizing the experimental fitting parameters in the literature, for material component layers in SOI and Ge photodetector structures. Furthermore, the transmittance, reflectance, absorbance and emissivity are calculated based on the obtained $n$ and $k$. By understanding the optical and electrical properties, their behavior, as function of thickness and photon energy (or wavelength) for these multilayers, is predicted. The results of these simulations can be used to engineer these multilayers for various applications in optoelectronics.

\section{Theoretical model}

\section{Forouhi-Bloomer dispersion equation for $k(E)$ and $n(E)$}

Spectroscopic ellipsometry (SE) is a well-established optical technique that is utilized to characterize the optical functions and other related properties by performing the measurement of the relative phase change of reflected and polarized light. ${ }^{7}$ Refractive index $(n)$ describes the behavior of light propagating through the medium, which is normally defined as $n=[c / v]$, where $c$ is the speed of light in vacuum and $v$ is the phase velocity of light in the medium. Extinction coefficient, $k$, characterizes the ability of the light beam to penetrate into the material. The Forouhi-Bloomer dispersion equation, ${ }^{6}$ applicable to the entire wavelength from radio-wave to EUV spectral range, is proposed as in Eq. (I) and Eq. (II) for $k(E)$ and $n(E)$, respectively. ${ }^{8,9}$ This dispersion model is consistent with the Kramers-Kronig analysis.

$$
k(E)=\sum_{j=1}^{P} \frac{A_{j} E}{E^{2}-B_{j} E+C_{j}}+\sum_{i=1}^{q} \frac{A_{i}\left(E-E_{g}\right)}{E^{2}-B_{i} E+C_{i}}
$$




$$
\begin{gathered}
n(E)=n(\infty)+\sum_{j=1}^{P} \frac{D^{\prime}{ }_{j} E+F_{j}{ }_{j}}{E^{2}-B_{j} E+C_{j}}+\sum_{i=1}^{q} \frac{D_{i} E+F_{i}}{E^{2}-B_{i} E+C_{i}} \quad \text { Eq. (II) } \\
\text { where } D_{i}=\frac{A_{i}}{Q_{i}}\left(E_{g}-\frac{B_{i}}{2}\right) ; \\
F_{i}=\frac{A_{i}}{Q_{i}}\left(C_{i}-\frac{E_{g} B_{i}}{2}\right) ; \\
Q_{j}=\left(C_{j}-\frac{B_{j}^{2}}{4}\right)^{1 / 2}=\hbar / 2 \tau_{j} \\
D^{\prime}{ }_{j}=-\frac{A_{j} B_{j}}{2 Q_{j}} ; \\
F^{\prime}{ }_{j}=\frac{A_{j} C_{j}}{Q_{j}} .
\end{gathered}
$$

The fitting parameter, $A_{i,}$ in Eq. (I) is a dimensionless contant, which represents the amplitude of transition based on the transition probability. The quantity, $B$, is the energy difference between the initial and final states involved in the electron transition. The quantity $C_{j}=\left[\left(B^{2} / 4\right)+\left(\hbar^{2} / 2 \tau_{j}\right)\right]$, where $\tau_{j}$ represents the lifetime of the $j$ th excited electron state. The number of terms $p$ and $q$ are taken as the quantity of peaks in $k(E)$.

\section{Emissivity and absorption coefficient}

After obtaining the extinction coefficient and refractive index from the experimental measurements of transmittance $[T(\lambda)]$ and reflectance $[R(\lambda)]$, they can be calculated exactly, irrespective of the number of layers or differences between the angles of incidence. These quantities are wavelength dependent. The expression for the absorption coefficient $(\alpha)$ of the multilayers is given by Eq. (III).

$$
\alpha=\frac{4 \pi k}{\lambda}
$$

where, $k$ is the extinction coefficient.

The optical transmittance, which is an effective and accurate pathway to obtain the optical constants below the absorption edge, can be measured or calculated by Eq. (IV),

$$
T(\lambda)=\exp (-\alpha t)
$$

where, $t$ is the thickness of the material. The reflectance can be obtained from Eq. (V) and Eq. (VI),

$$
R_{1}=\frac{(n-1)^{2}+k^{2}}{(n+1)^{2}+k^{2}}
$$

and

$$
R_{2}=\frac{(n-s)^{2}}{(n+s)^{2}}
$$

where, $n$ is the refractive index of the first layer, $s$ is the refractive index of the second layer and $k$ is the extinction coefficient. Emissivity $(\varepsilon)$ is a parameter that identifies the radiative properties of materials under conditions of thermal (temperature) equilibrium with the ambient. ${ }^{10}$ It can be obtained from reflectance $(R)$ and transmittance $(T)$ of a layer as in Eq. (VII). From this, the absorptance, and hence the emissivity, is determined using $A=1-[T+R]$.

$$
\varepsilon=\frac{(1-R)(1-T(\lambda))}{1-R \cdot T(\lambda)}
$$

\section{Case study}

\section{Silicon on Insulator}

In this study, the radiative properties of SOI structures, based on the earlier studies of Ravindra et $\mathrm{a}^{11}$ on SIMOX (Separation by IMplanted Oxygen) structures, with 200-nm Si/400-nm $\mathrm{SiO}_{2} / \mathrm{Si}-$ substrate, as the control group, are considered to demonstrate the validity of the proposed simulation model. In Figure 1A, A case study, based on $\mathrm{Li}$ et al $\mathrm{l}^{12}$ considering the thickness of $\mathrm{Si}$ as $1000 \mathrm{~nm}$ on top of a $2000 \mathrm{~nm}$ thick $\mathrm{SiO}_{2}$, is presented. Another case study is based on Badri et al ${ }^{13}$ with the thickness of $\mathrm{SiO}_{2}$ as $2000 \mathrm{~nm}$ with the top layer of Si varying in thickness from 75 to $325 \mathrm{~nm}$. The investigation of the detailed optical properties of SOI structures, based on these two case studies, are presented as a function of thickness and photon energy (or wavelength). The simulations are implemented in MATLAB using the fitting parameters based on Forouhi-Bloomer dispersion model for the refractive index and extinction coefficient of $\mathrm{Si}$ and $\mathrm{SiO}_{2}$ and are summarized in Table 1.

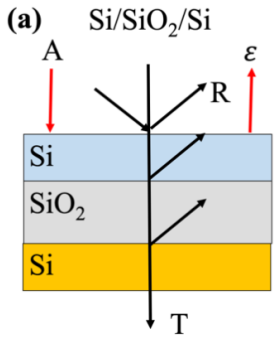

(c) $\mathrm{Si} / \mathrm{Ge} / \mathrm{Si} / \mathrm{SiO}_{2} / \mathrm{Si}$

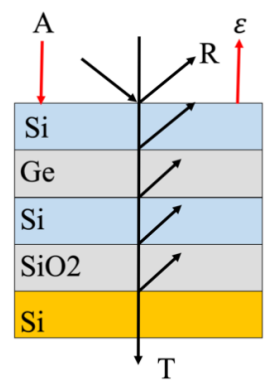

(b) $\mathrm{Si} / \mathrm{Ge} / \mathrm{Si}$

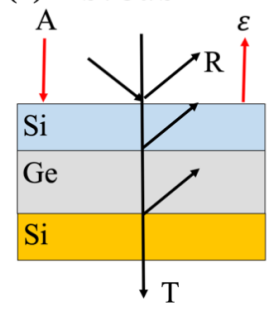

(d) $\mathrm{Si} / \mathrm{Ge} / \mathrm{Si} / \mathrm{Si} / \mathrm{SiO}_{2} / \mathrm{Si}$

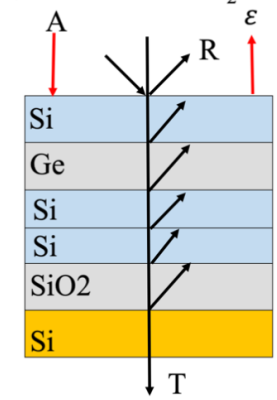

Figure I Schematic of (a) SOI, (b) $\mathrm{Si} / \mathrm{Ge} / \mathrm{Si}$, (c) $\mathrm{Si} / \mathrm{Ge} / \mathrm{SOI}$ and (d) $\mathrm{Si} / \mathrm{Ge} / \mathrm{Si} /$ sol Structures.

\section{Ge-on-Si photodetectors}

Here, we investigate the optical properties of several Ge based photodetectors, $\mathrm{Si} / \mathrm{Ge} / \mathrm{Si}$ and $\mathrm{Si} / \mathrm{Ge} / \mathrm{SOI}$, as shown in Figure $1 \mathrm{~b} \&$ 1c, as a function of thickness and photon energy (or wavelength). The thickness of Ge layer is varied from 400 to $1200 \mathrm{~nm}$ in steps of $200 \mathrm{~nm}$ (i.e., $400,600,800,1000,1200 \mathrm{~nm}$ ) and the thickness of bulk Si or SOI substrates consisting of thin $\mathrm{SiO}_{2}$ and silicon layer $(150 \mathrm{~nm} \mathrm{Si}$ on $400 \mathrm{~nm} \mathrm{SiO}_{2}$ decreasing to $60 \mathrm{~nm} \mathrm{Si}$ on $\left.150 \mathrm{~nm} \mathrm{SiO}_{2}\right)^{14}$ are considered. 
Besides, another interesting case study, based on Chong et al ${ }^{12}$ on coupled waveguides of Ge-on-SOI $\left(\mathrm{Si} / \mathrm{Ge} / \mathrm{Si} / \mathrm{Si} / \mathrm{SiO}_{2} / \mathrm{Si}\right)$, as shown in Figure 1d, with the thickness of Si (100nm), Ge (1000nm), Si(600 $\mathrm{nm}), \mathrm{Si}(220 \mathrm{~nm})$ and $\mathrm{SiO}_{2}(2000 \mathrm{~nm})$, respectively, is investigated. Besides the Ge photodetector, we also report the optical properties of
$\mathrm{Ge}_{\mathrm{x}} \mathrm{Si}_{1-\mathrm{x}}(\mathrm{x}=0.05,0.1$ and 0.15$)$ alloys on $\mathrm{Si}$ by varying the thickness of $\mathrm{Ge}_{\mathrm{x}} \mathrm{Si}_{1-\mathrm{x}}$ from $100-1000 \mathrm{~nm}$ based on the case study of Kadri et $\mathrm{al}^{15}$ The simulations are implemented in MATLAB using fitting parameters based on Forouhi-Bloomer dispersion model for the $n$ and $k$ of $\mathrm{Si}, \mathrm{Ge}, \mathrm{SiGe}$ and $\mathrm{SiO}_{2}$, as shown in Table 1.

Table I Fitting parameters of F-B dispersion model

\begin{tabular}{|c|c|c|c|c|c|c|}
\hline Experimental data & $\begin{array}{l}\text { Number of terms for } \\
\text { the calculations }\end{array}$ & $A_{i}(\mathrm{eV})$ & $B_{i}(e V)$ & $C_{i}(\mathrm{eV} 2)$ & $n(\infty)$ & $E_{g}(e V)$ \\
\hline \multirow[t]{4}{*}{ Si $(1.5-6 e V)^{9}$} & $q=4$ & 0.00405 & 6.885 & II.864 & 1.95 & 1.06 \\
\hline & & 0.01427 & 7.401 & 13.754 & & \\
\hline & & 0.0683 & 8.634 & 18.812 & & \\
\hline & & 0.17488 & 10.652 & $29.84 I$ & & \\
\hline \multirow[t]{10}{*}{$\mathrm{SiO}_{2}(0.03 \mathrm{I}-25 \mathrm{eV})^{6}$} & $p=5$ & $5.1915 \mathrm{E}-04$ & I.|489E-0I & $3.3119 E-03$ & 1.05 & \\
\hline & & I.59E-04 & $1.95 \mathrm{E}-0 \mathrm{I}$ & $9.53 \mathrm{E}-03$ & & \\
\hline & & $6.4532 \mathrm{E}-04$ & $2.699 \mathrm{E}-0 \mathrm{I}$ & I.8245E-02 & & \\
\hline & & 5.7594E-05 & $2.96 \mid 9 \mathrm{E}-0 \mathrm{I}$ & $2.195 \mathrm{IE}-02$ & & \\
\hline & & $2.5000 \mathrm{E}-0 \mathrm{I}$ & 0 & 3.8183 & & \\
\hline & $q=4$ & $5.7165 \mathrm{E}-02$ & $2.066 \mathrm{IE}+0 \mathrm{I}$ & $1.068 \mathrm{E}+02$ & & 9.0 \\
\hline & & I.4334E-0I & $2.3394 \mathrm{E}+0 \mathrm{I}$ & I.3749E+02 & & \\
\hline & & 4.3659E-02 & $2.723 \mathrm{IE}+0 \mathrm{I}$ & $1.8623 \mathrm{E}+02$ & & \\
\hline & & 2.1804 & $3.0863 \mathrm{E}+0 \mathrm{I}$ & $2.6368 \mathrm{E}+02$ & & \\
\hline & & 0.05366 & 21.940 & 125.443 & & \\
\hline \multirow[t]{4}{*}{$\mathrm{Ge}(0.5-7 \mathrm{eV})^{9}$} & $q=4$ & 0.00103 & 4.313 & 4.654 & 2.161 & 0.6 \\
\hline & & 0.11637 & 4.677 & 5.639 & & \\
\hline & & 0.10968 & 6.728 & 11.858 & & \\
\hline & & 0.03479 & 8.704 & 19.119 & & \\
\hline \multirow[t]{4}{*}{$\mathrm{Si}_{0.95} \mathrm{Ge}_{0.05}{ }^{14}$} & $P=I$ & 0.0022 & 6.8 & 11.7 & 1.79 & \\
\hline & $q=3$ & 0.083 & 7.3 & 14.1 & & 1.08 \\
\hline & & 0.012 & 8.6 & 18.7 & & \\
\hline & & 0.061 & 10.7 & 30.2 & & \\
\hline \multirow[t]{4}{*}{$\mathrm{Si}_{0.9} \mathrm{Ge}_{0.1}{ }^{14}$} & $P=I$ & 0.0061 & 6.7 & II.4 & 1.05 & \\
\hline & $q=3$ & 0.37 & 6.4 & 15.8 & & 1.05 \\
\hline & & 0.012 & 8.4 & 18.1 & & \\
\hline & & 0.13 & 10.5 & 38.4 & & \\
\hline \multirow[t]{4}{*}{$\mathrm{Si}_{0.85} \mathrm{Ge}_{0.15}{ }^{14}$} & $P=I$ & 0.014 & 6.6 & 11.2 & I.II & \\
\hline & $q=3$ & 0.7 & 5.3 & 15.1 & & 1.01 \\
\hline & & 0.02 & 8.5 & 18.2 & & \\
\hline & & 0.079 & 8.8 & 23.02 & & \\
\hline
\end{tabular}

\section{Results and discussion}

\section{Silicon on insulator}

The refractive index $(n)$ and extinction coefficient $(k)$, and their wavelength dependence, are the required fundamental properties for optical design of a variety of structures for applications in energy, optics and optoelectronics. These properties, as function of photon energy (or wavelength), for $\mathrm{Si}$ and $\mathrm{SiO}_{2}$, obtained from the ForouhiBloomer dispersion equation, are considered in these simulations. By inspecting the $n$ and $k$ spectra of each material, the entire dispersion pattern can be categorized into three distinct regions - near IR (NIR) energies $\left(10^{-2}\right.$ to $\left.1.0 \mathrm{eV}\right)$, NIR to UV energies $(1.0 \mathrm{eV}$ to $7 \mathrm{eV})$ and UV to EUV (Extreme UV) energies $(>7 \mathrm{eV})$. In Figure 2a, it is observed that $n$ of Si increases when the photon energy is $>1 \mathrm{eV}$, attaining a peak value of 6.7 at $3.5 \mathrm{eV}$; then, it decreases subsequently. $k$ of $\mathrm{Si}$ shows a similar dependence on photon energy as $n ; k$ increases when the energy is $>3.2 \mathrm{eV}$, reaching the peak value of 5.3 at $4.3 \mathrm{eV}$, then decreases to almost 0 . Both $n$ and $k$ of Si are consistent with the results of Forouhi et al. ${ }^{9}$ In Figure $2 \mathrm{~b}, n$ of $\mathrm{SiO}_{2}$ glass exhibits two peak values - one peak in the energy range of $0.06-0.16 \mathrm{eV}$ and another peak value of 2.45 at $10 \mathrm{eV}$, which may be due to the inter-band absorption. ${ }^{6} k$ of $\mathrm{SiO}_{2}$ has almost a similar pattern as $n$; the behavior of both $k$ and $n$, with photon energy, are consistent with those of Forouhi et al. ${ }^{6}$

In Figure 2c, the reflectance of Si has a high value of $>60 \%$ in the energy range of 3.2-5.6eV ( 220-400nm (UVA to UVC)). In Figure $2 \mathrm{~d}$, the reflectance of $\mathrm{SiO}_{2}$ exhibits several peaks with values $>20 \%$ from $0.06-5 \mathrm{eV}$. It can be concluded that the reflectance of $\mathrm{SiO}_{2}$ is highly related to the varying $n$, and the reflectance of $\mathrm{Si}$ is related to both $n$ and $k$. Several studies in the literature have confirmed the correlation of reflectance of materials with their surface roughness, measured 
using Atomic Force Microscopy; ${ }^{14}$ for example, Xie et al ${ }^{16}$ showed that the reflectance is strongly related to the surface morphology of three-dimensional germanium islands on $\mathrm{Si}(100)$ substrates. The dependence of the absorption coefficient of $\mathrm{Si}$ and $\mathrm{SiO}_{2}$, as function of energy, are shown in Figures $2 \mathrm{e} \& 2 \mathrm{f}$, respectively. Both of them have high values $>6 \times 10^{7} \mathrm{~cm}^{-1}$ for $\mathrm{E}>4 \mathrm{eV}$. In order to establish the validity of the simulations, in Figures 3a-3d, a case study of SIMOX, based on Ravindra et all ${ }^{11}$ has been considered (200-nm Si/ 400-nm $\left.\mathrm{SiO}_{2} / 700-\mu \mathrm{m} \mathrm{p}-\mathrm{Si}\right)$. In this Figure 3, the experimentally measured reflectance (Figure 3a), transmittance (Figure 3d), and emittance spectra (Figure $3 \mathrm{~b}$ ), of SIMOX are compared with the results from our modeling and simulations (Figure $3 \mathrm{c}$ ). A case study, based on Li et al ${ }^{12}$ with the thickness of top Si layer varying from 75-1000 nm on 2000 $\mathrm{nm} \mathrm{SiO}$ and $\mathrm{Si}$ as the substrate is simulated and presented in Figure 4. The results show that the SOI structures exhibit similar patterns with slight change in emissivity from $20 \%$ to $40 \%$ with the thickness of Si varied from $75 \mathrm{~nm}-1000 \mathrm{~nm}$. On the other hand, in Figure 5, SOI structures exhibit significant changes in emissivity, transmittance and reflectance in the wavelength range of $7-22 \mu \mathrm{m}$ when only the thickness of $\mathrm{SiO}_{2}$ is varied from 2000-500 nm. In the energy range of $0.06-0.21 \mathrm{eV}(\lambda=6-20 \mu \mathrm{m})$, the high emissivity of SOI is due to lattice vibrations in $\mathrm{SiO}_{2}$. Another case study is based on Badri et $\mathrm{al}^{13}$ with the thickness of $\mathrm{SiO}_{2}$ varying from $250-2000 \mathrm{~nm}$ and the top layer of Si varying from $125-1000 \mathrm{~nm}$. In Figure 6, the transmittance exhibits significant decrease from $>70 \%$ to $<10 \%$ and an increase in emissivity from $25 \%$ to $70 \%$ in the energy range of $0.06-0.18 \mathrm{eV}(\lambda=7-22 \mu \mathrm{m})$. Moreover, a peak in emissivity at $2.5 \mathrm{eV}(500 \mathrm{~nm})$ increases from $20 \%$ to almost $40 \%$ when the thickness of $\mathrm{Si}$ and $\mathrm{SiO}_{2}$ is increased from $125-1000 \mathrm{~nm}$ and $250-2000 \mathrm{~nm}$, respectively.
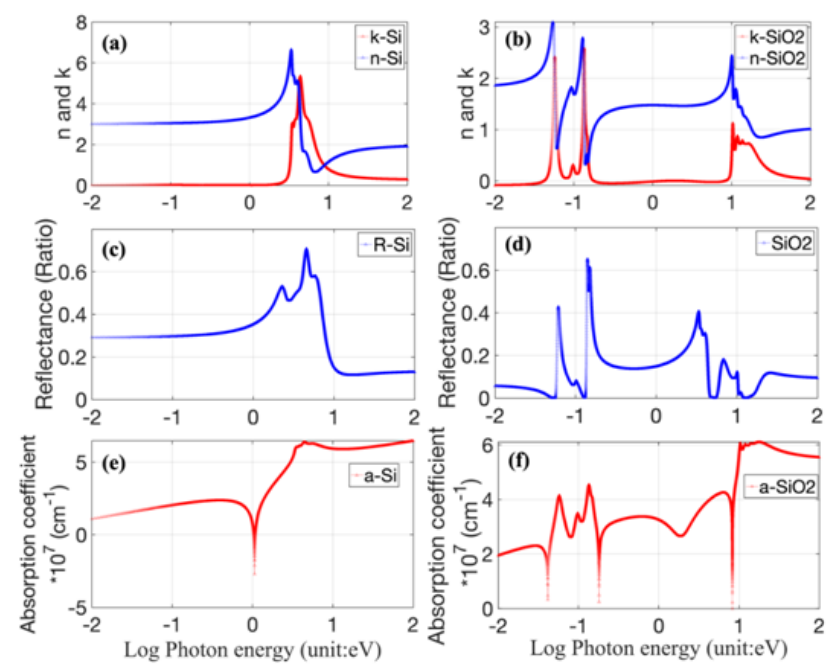

Figure 2 Simulation of $n$ and $k$ for (a) $\mathrm{Si}$ and (b) $\mathrm{SiO}_{2}$; optical reflectance of (c) $\mathrm{Si}$ and (d) $\mathrm{SiO}_{2}$; absorption coefficient of (e) $\mathrm{Si}$ and (f) $\mathrm{SiO}_{2}$ as function of energy.

Regardless of the varying thickness of $\mathrm{Si}$ and $\mathrm{SiO}_{2}$, the optical properties of SOI can be categorized into three parts - based on their spectral patterns. SOI exhibits relatively complicated changes in the radiative properties in the energy range of $0.04-0.18 \mathrm{eV}$. SOI exhibits $100 \%$ transmittance and $\sim 40 \%$ reflectance in the energy range of $0.13-1.58 \mathrm{eV}(\lambda=0.78-9.85 \mu \mathrm{m})$, and when the energy $<0.04 \mathrm{eV}$ $(\lambda>31 \mu \mathrm{m})$. The $\mathrm{Si} / \mathrm{SiO}_{2} / \mathrm{Si}$ structure shows $0 \%$ transmittance and over $90 \%$ reflectance and absorbance when the incident photon energy is $>3.16 \mathrm{eV}$. The thickness of the component layers has less influence in this photon energy range.
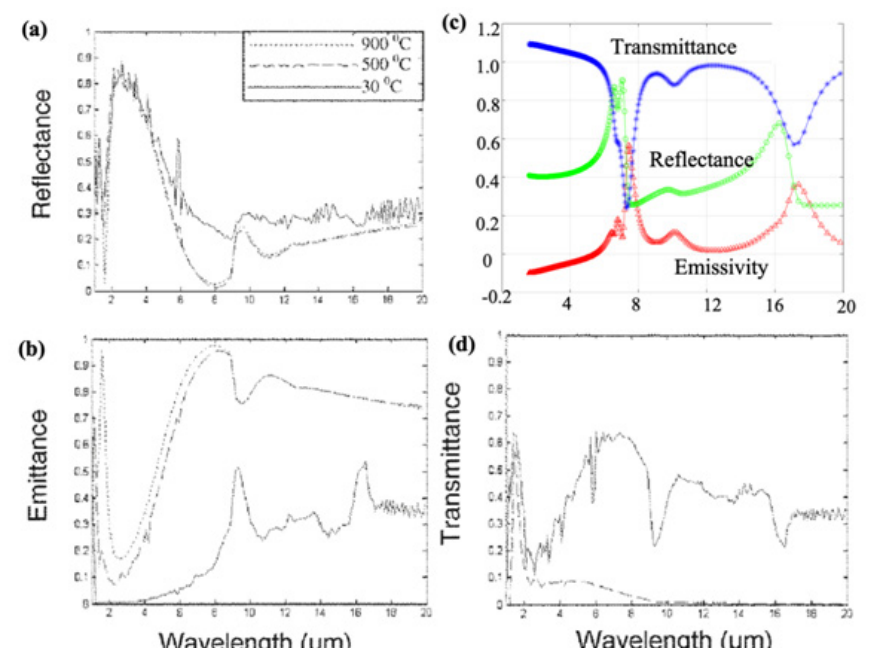

Figure 3 Comparison of optical properties of SIMOX - (a), (b), (d) measurements from the literature, at $30^{\circ} \mathrm{C}$, with simulations (c). Figure $3 \mathrm{a}, \mathrm{b}$ and $\mathrm{d}$ are reproduced with permission from Ref."

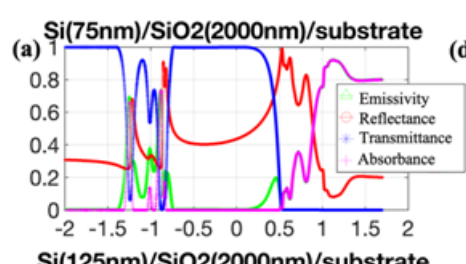

Si $(500 \mathrm{~nm}) / \mathrm{SiO} 2(2000 \mathrm{~nm}) /$ substrate
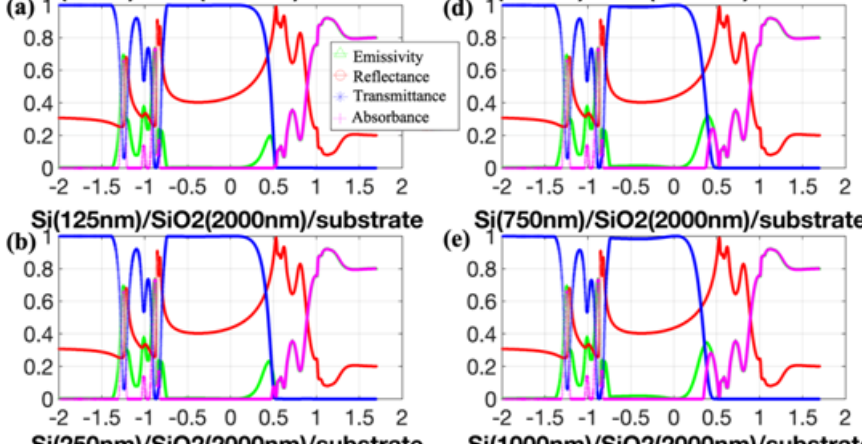

$\mathrm{Si}(750 \mathrm{~nm}) / \mathrm{SiO} 2(2000 \mathrm{~nm}) /$ substrate
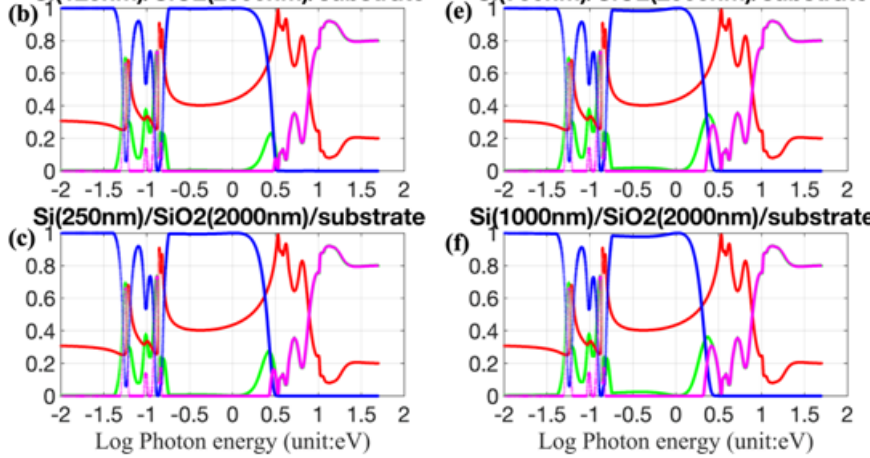

Figure 4 Emissivity, reflectance, transmittance and absorbance spectra of $\mathrm{Si} /$ $\mathrm{SiO}_{2}(2000 \mathrm{~nm}) / \mathrm{Si}$ structure as a function of photon energy and thickness.



Figure 5 Emissivity, reflectance, transmittance and absorbance spectra of $\mathrm{Si}$ $(1000 \mathrm{~nm}) / \mathrm{SiO}_{2} / \mathrm{Si}$ structure as a function of photon energy and thickness. 


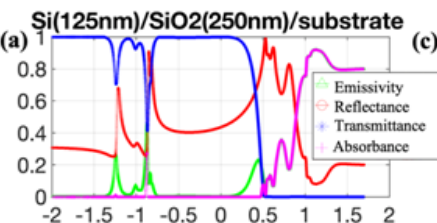

$\mathrm{Si}(500 \mathrm{~nm}) / \mathrm{SiO} 2(1000 \mathrm{~nm}) / \mathrm{substrate}$

(c)
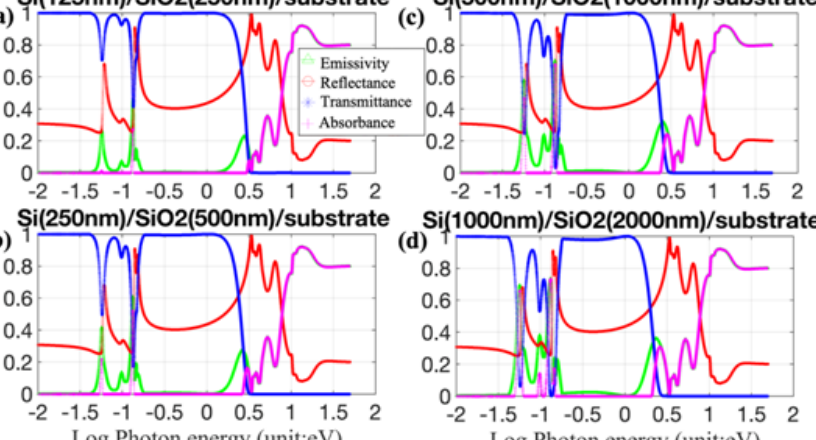

Figure 6 Emissivity, reflectance, transmittance and absorbance spectra of Si/ $\mathrm{SiO}_{2} / \mathrm{Si}$ structure as a function of photon energy and thickness.

\section{Ge-on-Si photodetectors}

Fundamental optical properties of $\mathrm{Si}, \mathbf{G e}$ and $\mathrm{SiGe}$ alloy: In Figure $7 \mathrm{a} \& 7 \mathrm{~b}, n$ and $k$ of $\mathrm{Si}$ and $\mathrm{Ge}$, as a function of energy, are presented. These spectral characteristics of $\mathrm{Si}$ and $\mathrm{Ge}$ are consistent with those of Forouhi et al. ${ }^{9}$ In Figure 7c, the reflectance of Si has a high value of $>60 \%$ in the energy range of $3.2-5.6 \mathrm{eV}(\lambda=220-400 \mathrm{~nm})$ (UVA to UVC). In Figure 7d, the reflectance of Ge exhibits several peaks with low reflectance in the energy range of $1-8 \mathrm{eV}$. Moreover, the absorption coefficient of $\mathrm{Si}$ and $\mathrm{Ge}$ have similar patterns and values $>5 \times 10^{7} \mathrm{~cm}^{-1}$ when the photon energy is $>4 \mathrm{eV}$. In Figure $8 \mathrm{a} \& 8 \mathrm{~b}, k$ of SiGe alloy shows similar dependence on wavelength to the $n$ of SiGe. The increase in $k$ at lower wavelengths $(\sim 400 \mathrm{~nm})$ is due to interband absorption, and a slow variation is observed when $\lambda$ is $>500$ $\mathrm{nm}$. In Figure 8c, the reflectance of SiGe starts with $20 \%$ for $\mathrm{SiGe}_{0.05}$; it increases to over $40 \%$ for $\mathrm{SiGe}_{0.15}$, when $\lambda$ is $\sim 350 \mathrm{~nm}$. However, a drastic decrease in reflectance, in the UV range $(\lambda=400-700 \mathrm{~nm})$, is observed when the Ge content is increased. These optical properties are consistent with those of Kadri et $\mathrm{al}^{14}$
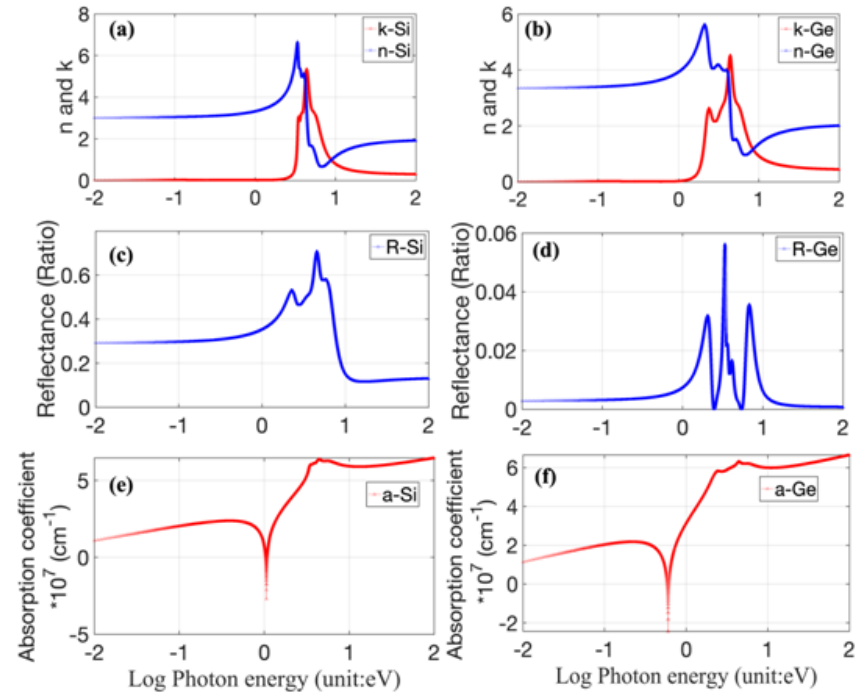

Figure 7 Simulation of $\mathrm{n}$ and $\mathrm{k}$ for (a) Si and (b) Ge; optical reflectance of (c) $\mathrm{Si}$ and (d) Ge; Absorption coefficient of (e) Si and (f) $\mathrm{Ge}$ as a function of photon energy.

Optical properties of $\mathrm{Si} / \mathrm{Ge} / \mathrm{Si}$ structure: The results of the simulation of the optical properties of $\mathrm{Si} / \mathrm{Ge} / \mathrm{Si}$ with varying thickness are shown in Figure 9. $\mathrm{Si} / \mathrm{Ge} / \mathrm{Si}$ multilayers are transparent for photon energy $<1 \mathrm{eV}$ and exhibit $>80 \%$ absorbance when the photon energy is $>8 \mathrm{eV}$. The changes in thickness of both $\mathrm{Si}$ and Ge have limited impact on the reflectance, emissivity, transmittance and absorbance. In Figure 9e-9h, slight changes (in 10\%) in absorbance (highlighted black circles) are observed.
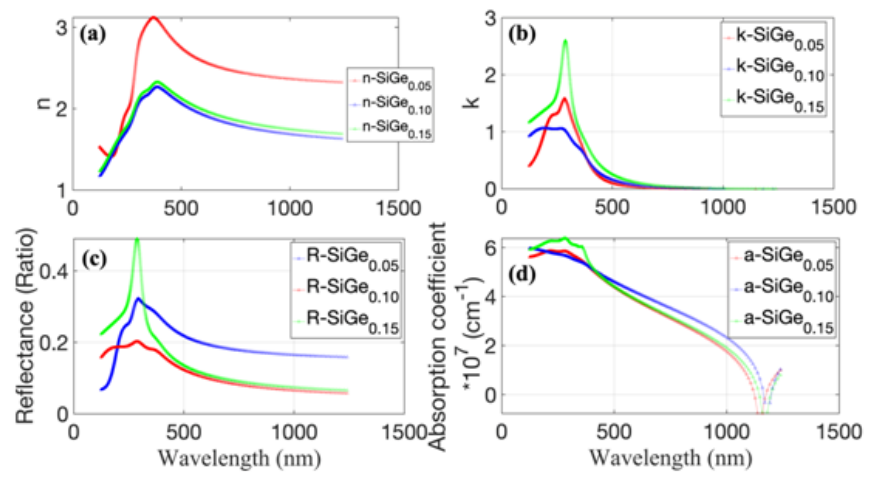

Figure 8 Simulation of optical properties of SiGe alloys - (a) n, (b) k, (c) optical reflectance and (d) absorption coefficient as a function of wavelength.
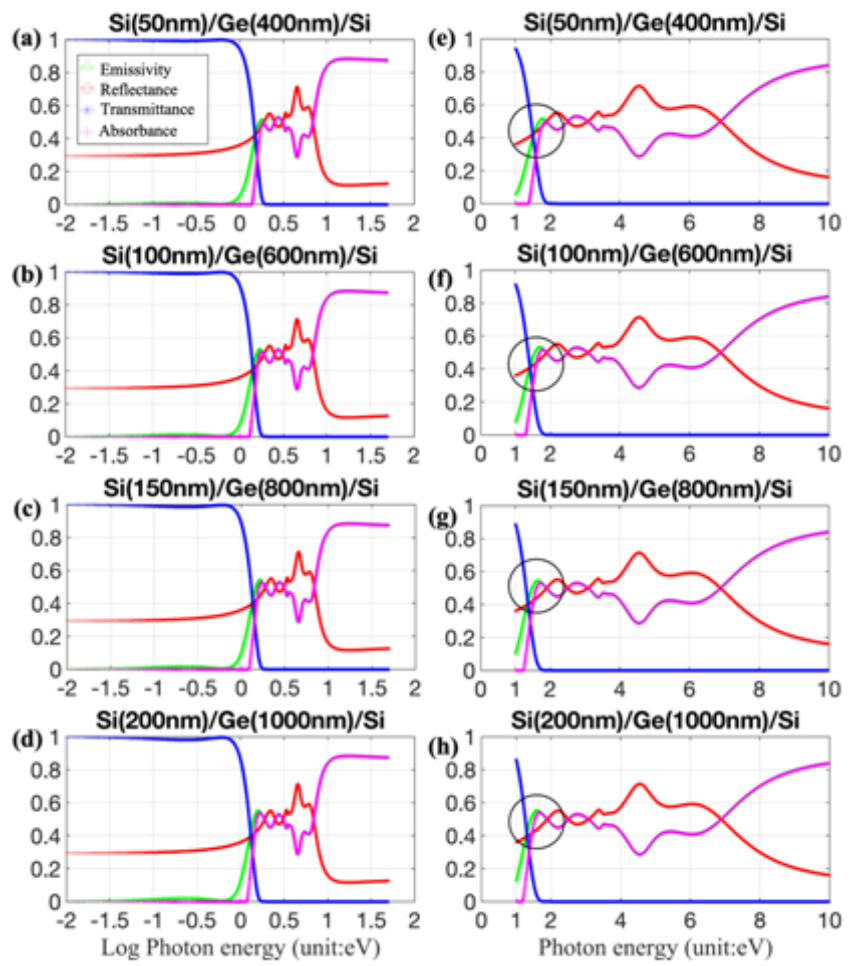

Figure 9 Emissivity, reflectance, transmittance and absorbance spectra of $\mathrm{Si} /$ $\mathrm{Ge} / \mathrm{Si}$ structure as a function of energy and thickness.

Optical properties of $\mathbf{S i} / \mathbf{G e} / \mathbf{S O I}$ structure: The results of the simulation of optical properties of $\mathrm{Si} / \mathrm{Ge} / \mathrm{SOI}$, with varying thickness, are shown in Figure 10a-10d. Figure 10d shows a higher decrease in transmittance and higher increase in emissivity than the other case studies for photon energy in the range of $0.06-0.18 \mathrm{eV}$. The structure exhibits transparency for photon energy $>0.18 \mathrm{eV}$. The results of the simulation of optical properties for $\mathrm{Si} / \mathrm{Ge} / \mathrm{Si} / \mathrm{SOI}$, with varying thickness, are shown in Figure 11a \& 11b. In these figures, an increase in emissivity and a decrease in transmittance, for photon energy in the range of $0.06-0.18 \mathrm{eV}$, is observed when the thickness of each of the layers is doubled. 


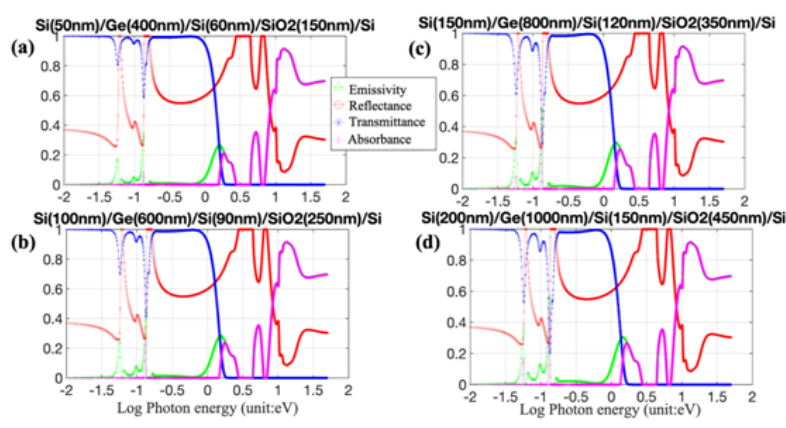

Figure I 0 Emissivity, reflectance, transmittance and absorbance spectra of $\mathrm{Si} /$ $\mathrm{Ge} / \mathrm{Si} / \mathrm{SiO}_{2} / \mathrm{Si}$ structure as a function of energy and thickness.
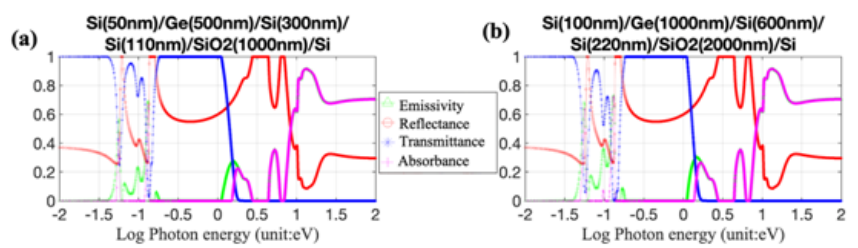

Figure II Emissivity, reflectance, transmittance and absorbance spectra, based on 2016, Chong et $\mathrm{al}^{12}$ as a function of energy and thickness.

Optical properties of SiGe/Si structure: The results of the simulation of optical properties of $\mathrm{SiGe} / \mathrm{Si}$, with varying thickness, are shown in Figure 12 . SiGe/Si shows almost $100 \%$ transmittance for $\lambda>600 \mathrm{~nm}$ when the Ge content is 0.05 and the thickness of SiGe is $100 \mathrm{~nm}$. The transmittance edge decreases when the Ge content is increased from 0.05 to 0.15 and the thickness of SiGe is increased from $100 \mathrm{~nm}$ to $1100 \mathrm{~nm}$. The reflectance increases slightly at $\lambda \sim 300 \mathrm{~nm}$ when the Ge content is increased from 0.05 to 0.15 . $\mathrm{SiGe}_{0.15}$ is more sensitive to the change in thickness compared with $\mathrm{SiGe}_{0.05}$, which exhibits almost a constant behavior in the optical properties, irrespective of the thickness. The absorbance decreases from over $90 \%$ to $0 \%$ in the wavelength range of 200-1200 $\mathrm{nm}$ with a slight rebound at $\sim 400$ -

\section{Conclusion}

In summary, this study presents a simulation of the wavelength dependence of the fundamental optical constants, the extinction coefficient and refractive index, based on the Forouhi-Bloomer dispersion equation. The simulation is applicable to the entire wavelength from radio waves to EUV spectral range. This model has been tested on various multilayers (SOI and Ge photodetector), and the results obtained are in good accord with some published data, as well as the literature. These models facilitate the calculation of the optical constants in a wide range of wavelengths. The efficacy of the proposed model is that it represents the correct picture of the optical and electronic properties depending on the structural evolution in the multilayers. Moreover, it is a well-established fact that the optoelectronic properties are susceptible to the refractive index and extinction coefficient of materials and thickness in these multilayers. Therefore, reliable data of the optical constants of semiconductors, particularly in the infrared range of wavelengths, are provided in this study. These results can be utilized for the determination and realization of the optical response, as a function of photon energy and thickness, of bulk as well as multi-layered semiconductors.

\section{Acknowledgments}

None.
$600 \mathrm{~nm}$; this rebound is highly sensitive to thickness, especially for $\mathrm{SiGe}_{0.15}$. The SiGe/Si multilayers exhibit constant $100 \%$ transmittance, $0 \%$ absorbance and $20 \%$ reflectance when $\lambda$ is $>\sim 800 \mathrm{~nm}$.
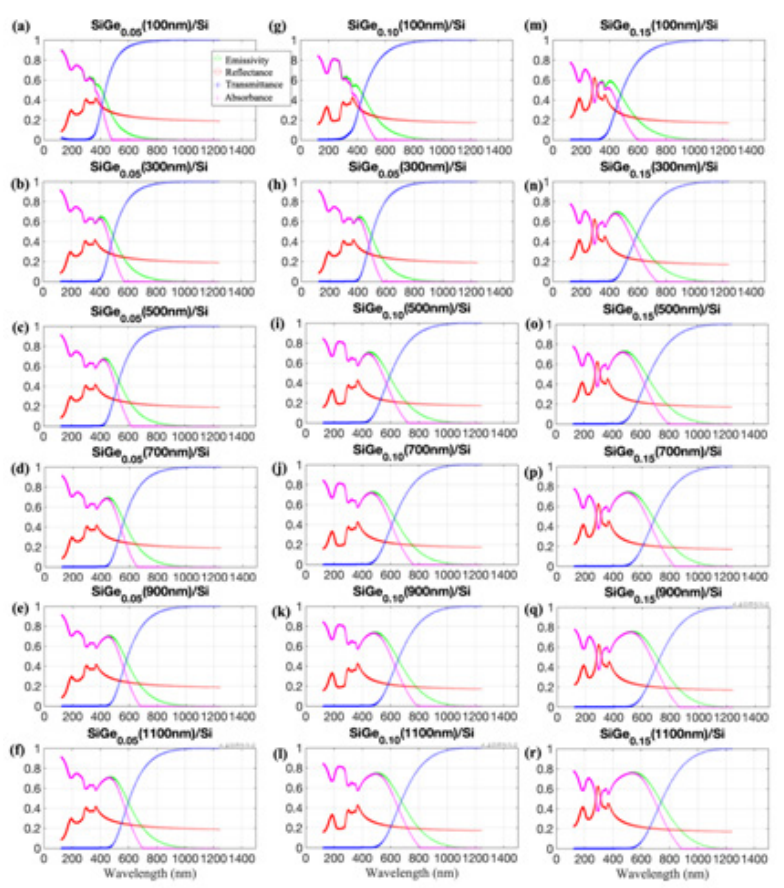

Figure 12 Emissivity, Reflectance, Transmittance and Absorbance spectra of $\mathrm{SiGe} / \mathrm{Si}$ as a function of wavelength and thickness.

As a matter of general interest in materials science, it may be noted that Demir et al ${ }^{17}$ have investigated the dispersion of the refractive index of poly(methyl methacrylate) (PMMA) and zinc oxide (zincite, $\mathrm{ZnO}$ ) nanoparticle composites using the phenomenological Cauchy relation. ${ }^{17}$ Accordingly, they have reported the Cauchy Coefficients of the films as a function of varying $\mathrm{ZnO}$ content in the PMMA-ZnO composites.

\section{Conflicts of interest}

The authors declare that there are no conflicts of interest.

\section{References}

1. Sun X. Germanium-on-Silicon for Integrated Silicon Photonics. In: Mohamed Fadhali, editor. Advanced Photonic Sciences. London, UK. IntechOpen; 2012.

2. Bonafos C, Paillard V, Jedrzejewski J, et al. Silicon nanocrystals embedded in oxide films grown by magnetron sputtering. AIMS Materials Science. 2016;3:538-561.

3. Michel J, Liu J, Kimerling LC. High-performance Ge-on-Si photodetectors. Nature Photonics. 2010;(8):527-534.

4. Fedeli JM, Orobtchouk R, Seassal C, et al. Integration issues of a photonic layer on top of a CMOS circuit. - art. no. 61250H. Proc SPIE. 2006:6125.

5. Liu J, Camacho-Aguilera R, Bessette JT, et al. Ge-on-Si optoelectronics. Thin Solid Films. 2012;520(8):3354-3360.

6. Forouhi AR, BloomerI. New dispersion equations for insulators and semiconductors valid throughout radio-waves to extreme ultraviolet spectral range. Journal of Physics Communication. 2019;3(3):035022.

7. Das SK, Khandaker J, Ahmed F. Simulation Study of Optical Properties of Transparent CdS Thin Film using the Sellmeier Empirical Dispersion Formula. International Journal of Photonics and Optical Technology. 2019;5(3):4-10. 
8. Forouhi R, Bloomer I. Optical dispersion relations for amorphous semiconductors and amorphous dielectrics. Physical review $B$ Condensed matter. 1986;34:7018-7026.

9. Forouhi R, Bloomer I. Optical properties of crystalline semiconductors and dielectrics. Physical review B Condensed matter. 1988;38:18651874.

10. Sopori BL, Chen W, Abedrabbo S, et al. Modeling emissivity of rough and textured silicon wafers. Journal of Electronic Materials. 1998;27(12):1341-1346.

11. Ravindra NM, Ravindra K, Mahendra S, et al. Modeling and simulation of emissivity of silicon-related materials and structures. Journal of Electronic Materials. 2003;32(10):1052-1058.

12. Li C, Xue C, Liu Z, et al. High-responsivity vertical-illumination Si/ Ge uni-traveling-carrier photodiodes based on silicon-on-insulator substrate. Scientific Reports. 2016;6(1):27743.
13. Hadi Badri S, Gilarlue MM, Gavgani SG. Ultra-thin silicon-oninsulator waveguide bend based on truncated Eaton lens implemented by varying the guiding layer thickness. Photonics and Nanostructures - Fundamentals and Applications. 2020;39:100766.

14. Fedeli JM, Di Cioccio L, Marris-Morini D, et al. Development of Silicon Photonics Devices Using Microelectronic Tools for the Integration on Top of a CMOS Wafer. Advances in Optical Technologies. 2008;2008:412518.

15. Kadri E, Krichen M, Elleuch S, et al. Optical properties of Si1-xGex/Si thin films. Optical and Quantum Electronics. 2016;48(7):352.

16. Xie YH, Samavedam SB, Bulsara M, et al. Relaxed template for fabricating regularly distributed quantum dot arrays. Applied Physics Letters. 1997;71(24):3567-3568.

17. Demir MM, Koynov K, Akbey U, et al. Optical Properties of Composites of PMMA and Surface-Modified Zincite Nanoparticles. Macromolecules. 2007;40:1089-1100. 